\title{
INSETOS AQUÁTICOS ASSOCIADOS A MACRÓFITAS SUBMERSAS COM DIFERENTES COMPLEXIDADES MORFOLÓGICAS
}

PEIRÓ, Douglas F. Professor doutor do Departamento de Ciências Biológicas e da Saúde do Centro Universitário de Araraquara - UNIARA. Laboratório de Biologia Aquática, Programa de Mestrado em

Desenvolvimento Regional e Meio Ambiente. Rua Carlos Gomes 1338, 14801-340, Araraquara/SP. E-mail: douglaspeiro@hotmail.com.

SAULINO, Hugo H.. Doutorando do Programa de Pós-Graduação em Ecologia e Recursos Naturais (PPGERN), UFSCar, Laboratório de Ecologia de Insetos Aquáticos (LEIA), Rodovia Washington Luís (SP-310), Km

235, 13565-905, São Carlos/SP. GORNI, Guilherme Rossi. Professor doutor do Departamento de Ciências Biológicas e da Saúde do Centro Universitário de Araraquara - UNIARA. Laboratório de Biologia Aquática,

Programa de Mestrado em Desenvolvimento Regional e Meio Ambiente.

CORBI, Juliano José. Professor doutor do Departamento de Hidráulica e Saneamento, EESC, USP, Av.

Trabalhador sãocarlense 400, CP 359, 13566-590. São Carlos/SP.

FIORAVANTE, Adrie Pool; AMARAL, Gabriele. Discentes egressos do Curso de Ciências Biológicas do Centro Universitário de Araraquara - UNIARA.

\section{RESUMO}

O objetivo deste estudo foi analisar a diversidade e a estrutura da comunidade de insetos aquáticos associadas à macrófitas aquáticas submersas com diferentes complexidades morfológicas com relação às estruturas foliares. As coletas ocorreram em períodos seco e chuvoso, no Ribeirão das Anhumas, Américo Brasiliense/SP. Foram analisadas quatro espécies de macrófitas submersas: Vallisneria sp., Eleocharis sp., Egeria najas e Ottelia sp. Aentomofauna coletada foi identificada até o nível de família, com exceção de Chironomidae, identificada até nível de tribo. A estrutura da comunidade foi analisada através dos índices de diversidade de Simpson, Equitabilidade de Pielou, participação relativa de categoria funcional e dominância de táxons. A composição faunística entre os diferentes períodos de coleta foi analisada por meio de uma n-MDS com índice de Morisita. A estrutura da comunidade de insetos associada a macrófitas, com diferentes morfologias estruturais, foi analisada por meio da similaridade, calculada pelo índice de Bray-Curtis. Foram identificadas 17 famílias pertencentes a cinco ordens, de um total de 1642 espécimes. A família Hydropsychidae (Trichoptera) apresentou eudominância (52,6\%), seguidas pela tribo Pentaneurini (Chironomidae) (13,8\%) e da família Trichoryithidae (Ephemeroptera) $(10 \%)$. Os resultados demonstram que não houve grandes variações da diversidade entre as espécies demacrófitas analisadas e períodos de coleta. Os grupos funcionais predadores e coletores foram predominantes. Aanálise de n-MDS indicou ausência de variação entre os períodos de coleta. A análise de similaridade indicou que as macrófitas E. najas e Otellia sp. possuem estrutura faunística similar, diferenciando-se das demais espécies analisadas. Os resultados demonstraram que a estrutura morfológica das macrófitas pode apresentar distintas estruturas de comunidades de insetos aquáticos.

Palavras-chave: Entomofauna aquática; Hidrófitas; Sistema lótico; Córrego de baixa ordem.

\section{AQUATIC INSECTS ASSOCIATED WITH SUBMERGED MACROPHYTES WITH DIFFERENT MORPHOLOGICAL COMPLEXITIES}

\section{Abstract}

The aim of the study was to analyze the diversity and community structure of aquatic insects associated with species of submersed macrophytes with different morphological complexity in relation to leaf structure. Sampling occurred in raining and dry periods at Ribeirão das Anhumas, Américo Brasiliense/SP. Four macrophytes were 
analyzed: Vallisneria sp., Eleocharis sp., Egeria najas and Ottelia sp. The entomological community was identified up to the family level, and at tribe level for Chironomidae. The community structure was analyzed using diversity indices of Simpson, Equitability evenness, relative participation of functional category and taxa dominance. The dispersion of faunal composition between the different collection periods was analyzed using a n-MDS with Morisita index. The structure of the insect community associated with macrophytes with different structural morphologies was analyzed using structural similarity calculated by the Bray-Curtis index. Seventeen families were identified from five orders, a total of 1642 specimens. The family Hydropsychidae (Trichoptera) presented eudominance $(52.6 \%)$, followed by the tribe Pentaneurini (Chironomidae) (13.8\%) and family Trichoryithidae (Ephemeroptera) (10\%). The results showed that there were no large diversity variations in the analyzed macrophyte species and the sampling periods. The predator and collector functional groups were predominant. The n-MDS analysis indicated the absence of seasonal variation and, the similarity analysis indicated that macrophyte E. najas and Otellia sp., presented similar fauna structure, differing from other analyzed species. The results demonstrated that the morphological structure of macrophytes may have different structures of aquatic insect communities.

KEYwORDs: Aquatic entomofauna; Hydrophytes; Lotic system; Low order stream.

\section{INTRODUÇÃO}

Os macroinvertebrados aquáticos compõem um dos componentes mais ricos e diversificados das comunidades de água doce, habitando tanto ambientes lênticos como lóticos. Esses organismos são importantes componentes dos sistemas aquáticos, pois servem de alimento para peixes e crustáceos, além de desempenhar um papel central na dinâmica de nutrientes, na transformação de matéria e no fluxo de energia do ecossistema (ESTEVES, 1998; BUENO et al., 2003). Os macroinvertebrados de ambientes lóticos são representados por vários filos, entre eles o filo Arthropoda, que inclui os insetos, os ácaros e os crustáceos (HAUER \& RESH, 1996). Os insetos destacam-se por sua diversidade e abundância, correspondendo a uma grande porcentagem dessa fauna (HYNES, 1970; LAKE, 1990). A distribuição dos insetos aquáticos está diretamente relacionada às características morfológicas dos rios (velocidade da correnteza, profundidade e tipo de substrato), às características químicas e físicas da água (oxigênio dissolvido, pH e temperatura) (CALLISTO, 2005), como também pela disponibilidade de recursos alimentares (RESH \& ROSEMBERG, 1984; MERRITT \& CUMMINS, 1996).

Entre os tipos de habitat mais propícios para as comunidades de insetos aquáticos, destacam-se os bancos de macrófitas aquáticas. Esses vegetais apresentam variadas estruturações, que os classificam em diferentes grupos: emergentes (enraizadas no sedimento do corpo hídrico, com as folhas voltadas para a parte aérea - acima da superfície); com folhas flutuantes (enraizadas no sedimento, com as folhas flutuando na superfície d'água); livres flutuantes (que tipicamente flutuam na superfície d'água sem enraizamento no sedimento; e submersas (plantas que crescem completamente submersas na coluna d'água e enraizadas ao substrato) (CHAMBERS et al., 2008). As características dessas plantas possibilitam seu estabelecimento nos mais diversos compartimentos dos sistemas lóticos, como nas margens e nos leitos dos córregos. A presença dessa comunidade vegetal nos ambientes aquáticos contribui para uma maior heterogeneidade de habitat (MARGALEF, 1983), e é fonte de alimento para diversas espécies (THOMAZ et al., 2008). As diferenças existentes entre os animais presentes nas plantas estão relacionadas à procura de alimento e abrigo, como também à maior superfície fornecida pelas mesmas (MINSHALL, 1984; CORREIA, 1999). Alguns estudos afirmam que a riqueza e diversidade ecológica de invertebrados em sistemas aquáticos se correlacionam positivamente com a complexidade morfológica das plantas (THOMAZ 
et al., 2008; THOMAZ \& CUNHA, 2010), tendo em vista uma maior disponibilidade de nichos para a fauna associada.

A entomofauna fitófila é composta por ninfas, larvas e indivíduos adultos das mais diversas ordens. Entre os grupos de insetos aquáticos associados, destacamse os insetos minadores folheares (WARD, 1992), coletores detritívoros (PEIRÓ \& ALVES, 2006) e organismos casuais ou facultativos; poucos são os animais herbívoros que utilizam o tecido vegetal como fonte de alimento (SONODA, 1999). Muitos estudos são encontrados na literatura científica tratando de insetos aquáticos associados a macrófitas aquáticas de ambientes lênticos, (GLOWACKA et al., 1976; MASTRANTUONO, 1986; CORREIA, 1999; SONODA; 1999, CORBI \& TRIVINHOSTRIXINO, 2002; PEIRÓ \& ALVES, 2006. PEIRÓ \& GORNI, 2010); entretanto, estudos que abordam a associação de insetos aquáticos fitófilos em ambientes lóticos permanecem escassos (OLIVEIRA et al., 1997; AYRES-PERES et al., 2006).

Devido à importância desses organismos, que constituem um dos elos das cadeias de detritos e de herbívora no meio aquático, e também devido ao baixo conhecimento das suas relações com macrófitas em sistemas lóticos, o objetivo deste estudo foi analisar a diversidade e estrutura da comunidade de insetos aquáticos associada a macrófitas submersas com diferentes complexidades morfológicas em um córrego na região central do Estado de São Paulo. Para isso foi testada a seguinte hipótese: macrófitas submersas com diferentes complexidades morfológicas apresentam diversidade e estrutura de comunidades de insetos diferenciadas.

\section{Material e MÉtodos}

\section{Área de estudo}

As coletas ocorreram em um trecho do Ribeirão das Anhumas (Américo Brasiliense/SP), situado na região central do Estado de São Paulo. Este ribeirão é classificado de $1 .^{\mathrm{a}}$ a 3 . $^{\mathrm{a}}$ ordem, com água bem oxigenada, pertencente à bacia hidrográfica do rio Mogiguaçu. A amostragem das macrofitas foi realizada em um canal de escoamento de uma represa $\left(21^{\circ} 42^{\prime} 18^{\prime \prime S}\right.$, $048^{\circ} 01^{\prime} 32^{\prime \prime} \mathrm{O}$, altitude $534 \mathrm{~m}$ ) pertencente a um clube de atividades náuticas. Esse segmento do ribeirão apresenta largura média de 3 metros, com profundidade média de 1 metro e substrato arenoso.

\section{Método de coleta}

As coletas ocorreram em dois períodos de seca e de chuva: 30 de junho de 2007 e 5 de abril de 2008 (períodos secos), 6 de outubro de 2007 e 18 de janeiro de 2008 (períodos chuvosos). Foram selecionadas quatro espécies de hidrófitas submersas com estruturas morfológicas diferentes (Figura 1), localizadas no leito do córrego: Vallisneria sp., Eleocharis sp. (gramínea anfíbia) - classificadas com estrutura morfológica simples, tendo em vista as folhas do tipo lanceoladas e não ramificadas; Egeria najas, e Ottelia sp. classificadas como macrófitas de estrutura morfológica complexa, apresentando maior número de inserções folheares. As plantas foram removidas manualmente, com auxílio de uma rede "D" de malha com abertura de $0,25 \mathrm{~mm}$. O material coletado foi transferido para galões de 5 litros contendo 1,5 litros de água do local. Após a amostragem, os recipientes foram transportados para o laboratório para a triagem e separação da entomofauna associada às plantas. 


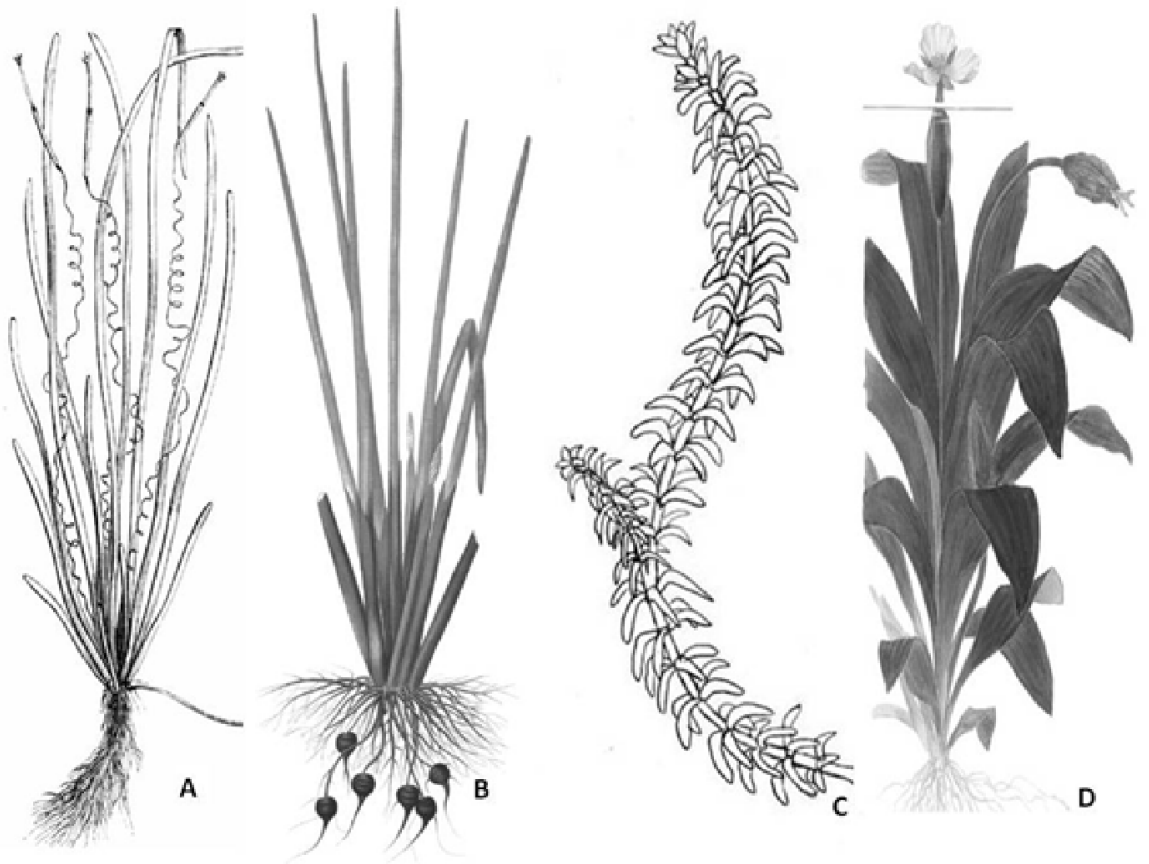

Figura 1 - Macrófitas aquáticas submersas com diferentes estruturas morfológicas coletadas no Ribeirão das Anhumas, Américo Brasiliense/SP. Estrutura morfológica simples: A. Vallisneria sp. e B. Eleocharis sp.

Estrutura morfológica complexa: C. Egeria najas e D. Ottelia sp.

Fonte: Ilustração adaptada e modificada de: http://www.nhn.leidenuniv.nl/, http://www.plantsystematics.org/ e http://www.idtools.org.

\section{Triagem e identificação da entomofauna}

As larvas de insetos aquáticos foram triadas em bandejas transiluminadas e identificadas como auxílio de lupas até o nível de família, com exceção de Chironomidae (identificados até nível de tribo), utilizando os seguintes guias de identificação: Mccfferty (1981), Merritt \& Cummins (1996), Nieser \& Melo (1997), Carvalho \& Calil (2000) e Fernandez \& Dominguez (2001). As categorias funcionais de alimentação dos insetos coletados foram estabelecidas de acordo a classificação adotada por Merrit \& Cummins (1996), considerando as seguintes categorias: fragmentadores (que se alimentam de grandes partículas de matéria orgânica morta), raspadores (que se alimentam de algas perifíticas), coletores (que se alimentam de matéria orgânica morta de partículas finas) e predadores (que se alimentam das demais categorias funcionais, e outros animais).

\section{Análise dos dados}

A estrutura da comunidade de insetos aquáticos em cada macrófita foi analisada através dos índices de diversidade de Simpson, Equitabilidade de Pielou e da participação relativa de cada categoria funcional de alimentação. A dominância dos táxons foi estabelecida de acordo com suas relativas abundância: eudominantes $(>10 \%)$, dominantes $(10 \%)$, subdominantes $(2-5 \%)$, recessivos (1-2\%), subrecessivos $(<1 \%)$ (de acordo com Trivinho-Strixino, 1993). Após a identificação e contagem, os espécimes foram armazenados em frascos de vidro etiquetados contendo ETOH 75\% e depositados na coleção do Laboratório de Biologia Aquática do Departamento de Ciências Biológicas e da Saúde do Centro Universitário de Araraquara - UNIARA.

Visando obter uma relação de dispersão da composição da fauna em relação às estações sazonais 
(seca e chuvosa), foi obtido um diagrama de ordenação resultante de um escalonamento multidimensional não métrico (n-MDS) com índice de Morisita. Para essa análise foram utilizados dados brutos de abundância de todas as coletas correspondentes às estações sazonais, obtidas por meio da análise das macrófitas aquáticas.

A análise de agrupamento (UPGMA) de Bray Curtis com os dados de abundância foi empregada com o objetivo de verificar a similaridade na composição da fauna bentônica dentre as macrófitas estudadas. Para a realização das análises foi utilizado o software Palaeontological Statistics (PAST - versão 1.49) (HAMMER et al., 2001).

\section{Resultados}

Foram identificadas 17 famílias pertencentes a cinco ordens, em um total de 1.642 espécimes (Tabela 1). Analisando a composição da entomofauna associada às macrófitas, observou-se que a Família Hydropsychidae (Trichoptera) foi a mais abundante, apresentando eudominância $(52,6 \%)$, seguida da tribo Pentaneurini (Chironomidae) $(13,8 \%)$ e da Família Trichoryithidae (Ephemeroptera) (10\%). Outras famílias da ordem Trichoptera - Hydroptilidae, Odontoceridae e Leptoceridae - apresentaram dominância e subdominância durante os dois períodos, indicando uma expressiva participação relativa nos períodos de coleta. Outras famílias se apresentaram variando entre subdominância e recessividade, porém muitos dos táxons identificados apresentaram baixa participação relativa (sub-recessivos) (Tabela 1). O período chuvoso apresentou a maior abundância de insetos dentre os dois períodos coletados. As menores abundâncias associadas às macrófitas foram obtidas em Vallisneria sp. e Ottelia sp., no período seco, e em $E$. najas, no período chuvoso.

Tabela 1 - Composição taxonômica e classificação de dominância dos insetos aquáticos associados a macróficas submersas com diferentes estruturas morfológicas, no Ribeirão das Anhumas, região centraldo

Estado de São Paulo. Pr: predador, Co: coletor, Ra: raspador, Fr: fragmentador.

\begin{tabular}{|c|c|c|c|c|c|c|c|c|c|c|}
\hline \multirow{4}{*}{$\begin{array}{c}\text { Ordem } \\
\text { Ephemeroptera }\end{array}$} & \multirow{5}{*}{\begin{tabular}{|l|}
\multicolumn{1}{c|}{ Taxa } \\
Tricorythidae \\
Ephemerellidae
\end{tabular}} & \multirow{4}{*}{$\begin{array}{c}\begin{array}{c}\text { Categoria } \\
\text { Funcional }\end{array} \\
\mathrm{Co}\end{array}$} & \multirow{2}{*}{\multicolumn{4}{|c|}{\begin{tabular}{|c|} 
Período Seco \\
Macrófita
\end{tabular}}} & \multirow{2}{*}{\multicolumn{4}{|c|}{$\begin{array}{c}\text { Período Chuvoso } \\
\text { Macrófita }\end{array}$}} \\
\hline & & & & & & & & & & \\
\hline & & & \multirow{2}{*}{\begin{tabular}{|c|} 
Vallesneria \\
1
\end{tabular}} & \multirow{2}{*}{$\begin{array}{c}\text { Eleocharis } \\
28\end{array}$} & \multirow{2}{*}{$\begin{array}{c}\text { Egeria } \\
27\end{array}$} & \multirow{2}{*}{$\frac{\text { Ottellia }}{37}$} & \multirow{2}{*}{\begin{tabular}{|c|} 
Vallesneria \\
13
\end{tabular}} & \multirow{2}{*}{$\begin{array}{c}\text { Eleocharis } \\
48\end{array}$} & \multirow{2}{*}{$\begin{array}{l}\text { Egeria } \\
0\end{array}$} & \multirow{2}{*}{$\begin{array}{l}\text { Ottellia } \\
9\end{array}$} \\
\hline & & & & & & & & & & \\
\hline \multirow{5}{*}{ Diptera } & & Co & 0 & 0 & 2 & 0 & 0 & 10 & 0 & 0 \\
\hline & Leptohyphidae & Co & 0 & 21 & 13 & 2 & 0 & 13 & 28 & 32 \\
\hline & Empididae & $\operatorname{Pr}$ & 0 & 0 & 0 & 0 & 3 & 0 & 1 & 1 \\
\hline & Chironomini & Co & 0 & 2 & 2 & 2 & 0 & 4 & 2 & 0 \\
\hline & Tanytarsini & Co & 2 & 0 & 0 & 0 & 9 & 0 & 0 & 1 \\
\hline \multirow{5}{*}{ Odonata } & Pentaneurini & $\operatorname{Pr}$ & 15 & 11 & 52 & 5 & 48 & 81 & 2 & 12 \\
\hline & Orthocladiinae & Co & 0 & 3 & 3 & 1 & 2 & 9 & 4 & 3 \\
\hline & Calopterygidae & $\operatorname{Pr}$ & 2 & 3 & 1 & 2 & 1 & 0 & 4 & 2 \\
\hline & Protoneuridae & $\operatorname{Pr}$ & 0 & 1 & 1 & 0 & 0 & 0 & 0 & 0 \\
\hline & Libellulidae & $\operatorname{Pr}$ & 1 & 2 & 0 & 2 & 0 & 1 & 0 & 0 \\
\hline \multirow{4}{*}{ Trichoptera } & Coenagrionidae & $\operatorname{Pr}$ & 0 & 1 & 0 & 0 & 0 & 3 & 0 & 0 \\
\hline & Hydropsychidae & Co & 3 & 84 & 192 & 2 & 150 & 91 & 97 & 245 \\
\hline & Hydroptilidae & $\mathrm{Ra}$ & 10 & 0 & 39 & 0 & 8 & 9 & 0 & 2 \\
\hline & Odontoceridae & $\mathrm{Fr}$ & 0 & 5 & 0 & 6 & 0 & 8 & 3 & 13 \\
\hline \multirow{3}{*}{ Coleoptera } & Leptoceridae & $\mathrm{Fr}$ & 0 & 0 & 6 & 5 & 4 & 22 & 22 & 12 \\
\hline & Hydrophilidae & $\operatorname{Pr}$ & 0 & 1 & 1 & 0 & 4 & 6 & 0 & 1 \\
\hline & Total & & 34 & 162 & 339 & 64 & 242 & 305 & 163 & 333 \\
\hline
\end{tabular}

Fonte: Dados de pesquisa. 
A análise da estrutura da comunidade indicou que não houve grandes alterações das variáveis comunitárias dentre as diferentes macrófitas, como também dentre os diferentes os períodos de amostragem. As maiores diversidades estiveram presentes em Vallisneria sp. (no período seco) e em Eleocharis sp. (no período chuvoso). Os menores índices de diversidade foram observados em E. najas (no período seco) e Ottelia sp. (no período chuvoso). $\mathrm{O}$ mesmo resultado foi obtido com o índice de equitabilidade de Pielou (Tabela 2).
Os grupos funcionais de alimentação predadores e coletores predominaram entre as diferentes macrófitas e períodos de coleta. E. najas e Ottelia sp. apresentaram menores participações relativas de predadores no período chuvoso. Vallisneria sp. e E. najas apresentaram maior participação relativa do grupo de insetos raspadores no período seco. A participação relativa do grupo de insetos fragmentadores foi maior nas macrófitas E. najas e Eleocharis sp. (no período chuvoso), seguido de Ottelia sp. nos períodos (seco e chuvoso) (Tabela 2).

Tabela 2. Análise da estrutura das comunidades de insetos aquáticos associados a macrófitas submersas com diferentes estruturas morfológicas, no Ribeirão das Anhumas, região central do Estado de São Paulo.

\begin{tabular}{l|cccc|cccc}
\hline & \multicolumn{4}{|c|}{ Périodo seco } & \multicolumn{4}{c}{ Périodo chuvoso } \\
\cline { 2 - 9 } & Vallisneria & Eleocharis & Egeria & Otellia & Vallisneria & Eleocharis & Egeria & Otellia \\
\hline Riqueza_S & 7 & 12 & 12 & 10 & 10 & 13 & 9 & 12 \\
Abundância & 34 & 162 & 339 & 64 & 242 & 305 & 163 & 333 \\
Dominância & 0,30 & 0,32 & 0,37 & 0,36 & 0,43 & 0,20 & 0,40 & 0,56 \\
Simpson_1-D & 0,70 & 0,68 & 0,63 & 0,64 & 0,57 & 0,80 & 0,60 & 0,44 \\
Equitabilidade_J & 0,76 & 0,62 & 0,57 & 0,67 & 0,55 & 0,76 & 0,58 & 0,43 \\
Coletores (\%) & 17,65 & 85,19 & 70,50 & 75,86 & 72,80 & 57,38 & 80,86 & 87,61 \\
Predadores (\%) & 52,94 & 11,73 & 16,22 & 15,52 & 22,18 & 29,84 & 3,70 & 4,23 \\
Raspadores (\%) & 29,41 & 0,00 & 11,50 & 0,00 & 3,35 & 2,95 & 0,00 & 0,60 \\
Fragmentadores (\%) & 0,00 & 3,09 & 1,77 & 8,62 & 1,67 & 9,84 & 15,43 & 7,55 \\
\hline
\end{tabular}

Fonte: Dados de pesquisa.

A análise n-MDS confirma a ausência de variação sazonal da entomofauna em relação às macrófitas amostradas, visto que não foi observada nítida separação entre as estações seca de chuvosa (Figura
2). A tendência de agrupamento das amostras na região central do gráfico indica a proximidade dos valores de abundância registrados em cada uma delas. 


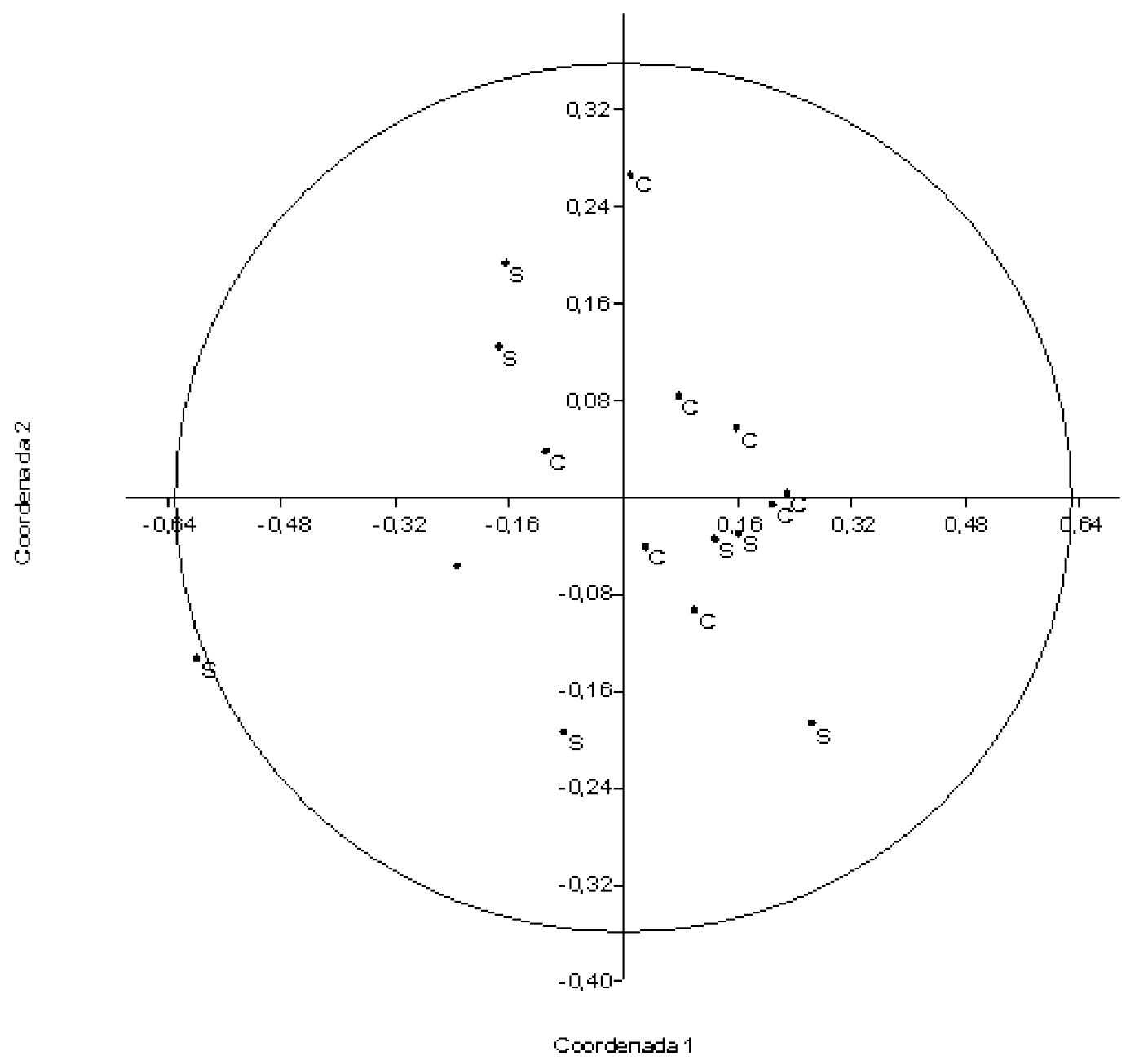

Figura 2 - n-MDS das amostras de macrófitas coletadas em diferentes períodos sazonais no Ribeirão das Anhumas (Américo Brasiliense/SP). S: estação seca; C: estação chuvosa. Valor do stress = 0,1465. Fonte: Dados de pesquisa.

A análise de agrupamento indicou os gêneros de macrófitas E. najas e Otellia sp. como os mais similares (aproximadamente $80 \%$ de similaridade) (Figura 3). Em contrapartida, a macrófita Valisneria sp. apresentou composição faunística menos similar, com cerca de $64 \%$ de similaridade. Ademais, foi possível verificar, de acordo com a análise, uma nítida separação de três grupos (1: E. najas e Otellia sp.; 2: Eleocharis sp.; 3: Valisneria sp.) refletindo diferentes complexidades morfológicas das macrófitas em questão. 

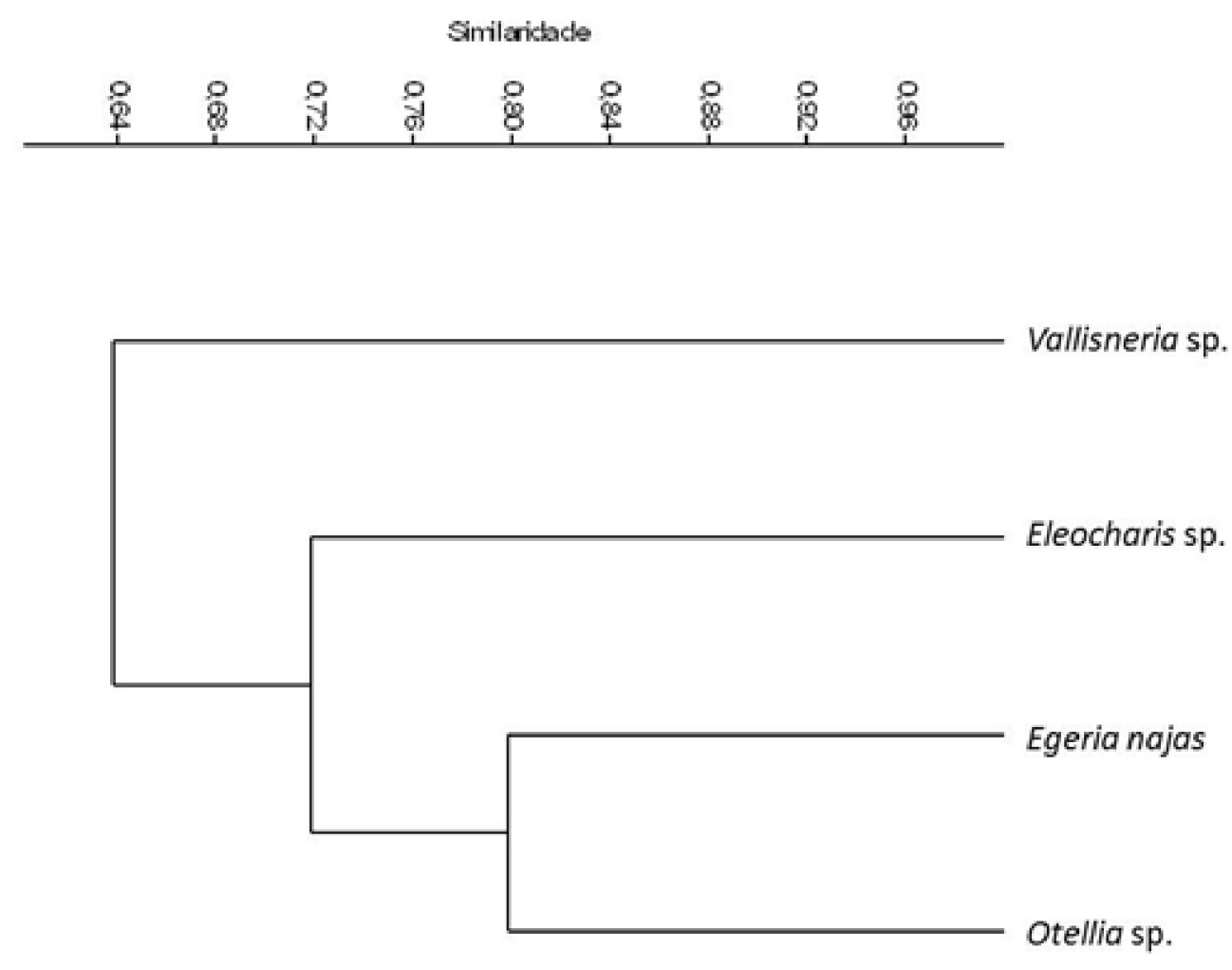

Figura 3 - Análise de similaridade da entomofauna associada à macrófitas aquáticas coletadas no Ribeirão das Anhumas (Américo Brasiliense/SP). Correlação cofenética =0,91.

Fonte: Dados de pesquisa.

\section{Discussão}

As macrófitas aquáticas possibilitam o acúmulo de detritos, favorecendo a associação de uma rica fauna (DUDGEON, 1988; BLANCO-BELMONTE 1989/ 90; TRIVINHO-STRIXINO \& STRIXINO 1993). E a baixa variação da diversidade e riqueza observada no local de estudo se deve mais aos hábitos alimentares ou necessidade de abrigo dos insetos (WARD, 1992; CORREIA, 1999).

Diversos autores têm reportado a família Hydropsychidae como a mais abundante entre os Trichoptera nos ecossistemas lóticos (UIEDA \& GAJARDO, 1996; BISPO \& OLIVEIRA, 1998). A predominância dessa família, classificada como coletora, pode estar relacionada com algumas variáveis do córrego. A presença de correnteza e baixa profundidade junto aos bancos de macrófitas proporcionou um local favorável para a colonização, contribuindo com uma maior disponibilidade de alimento, composto principalmente por matéria orgânica morta de partículas finas. Outra característica está relacionada à biologia dos Hydropsychidae, que costumam obter alimento através da construção de casulos fixados em rochas, galhos ou plantas (WIGGINS, 1977; OLIVEIRA \& FROEHLICH, 1996), o que pode ter favorecido a dominância dessa família entre os bancos de macrófitas.

As categorias funcionais de alimentação mais abundantes no presente estudo nos leva a supor que o tecido vegetal vivo seja muito pouco utilizado, em decorrência da considerável participação de coletores e de predadores. Este resultado está de acordo com 
outros autores, que têm demonstrado que o tecido de uma macrófita aquática seja pouco utilizado como alimento. Porém, é mais comum que espécies ditas como minadoras folheares a utilizem para obtenção de oxigênio (BOYD, 1970; GLOWACKA et al, 1976; LAMBERTI \& MOORE, 1984; STRIXINO \& TRIVINHO-STRIXINO, 1984; ROSINE, 1955). Nesse caso, a presença de macrófitas no leito do córrego pode ter desempenhado um papel semelhante a um filtro acumulador de detritos, proporcionando à fauna um local que propicia a obtenção de alimento.

A ausência de distinção entre os períodos de coleta pode também ser explicada pela utilização dos bancos de macrófitas como habitat, onde a entomofauna encontra proteção contra predadores, como, por exemplo, de pequenos peixes. Em alguns estudos, observou-se que variação de fluxo d'água entre essas duas estações pode gerar maior delimitação de habitat nos sistemas lóticos (BROW \& BRUSSOCK, 1991; AGRANDI, 1996; SAULINO et al., 2011). No período seco, com o fluxo d'água menor, há maior distinção e separação de pontos de corredeiras e remansos, enquanto no período chuvoso a tendência é ocorrer uma maior homogeneização de habitat, devido às inundações. Os resultados indicam que a entomofauna presente nos bancos de macrófitas não foi afetada por essa variável ambiental.

A maior similaridade entre os táxons de insetos aquáticos registrados nas macrófitas E. najas e Ottelia sp. está relacionada com suas estruturas morfológicas, classificadas neste estudo como mais complexas em relação as demais espécies estudadas. Diversos estudos registram uma relação positiva entre a complexidade estrutural desses vegetais com a diversidade de insetos (THOMAZ et al., 2008; THOMAZ \& CUNHA, 2010). A presença de maior quantidade de inserções folheares pode ter proporcionado maior quantidade de nichos, abrigando assim um grupo entomofaunístico mais semelhante.

Os resultados demonstram a capacidade potencial de as macrófitas aquáticas abrigarem uma considerável riqueza e abundância de insetos aquáticos, e que suas complexidades morfológicas podem apresentar distintas estruturas de comunidades.

Agradecimentos

Este estudo é parte do trabalho de conclusão de curso de APF e GA. Agradecemos a identificação das macróficas, feitas gentilmente pelo Dr. Claudinei da Cruz, Dr. Robinson Antonio Pitelli e Dra. Silvia Patricia Carraschi. Agradecemos todo o suporte na aquisição do Laboratório de Biologia Aquática, pela coordenação do Curso de Biologia da UNIARA, na pessoa da profa. Teresa K. Muraoka; e pela da chefia do Departamento de Ciências Biológicas e da Saúde, na pessoa da Profa. Dra. Celi. V. Crepaldi.

\section{REFERÊNCIAS}

ANGRADI, T. R. Inter-habitat variation in benthic community structure, function, and organic matter storage in three Appalachian headwater streams.

\section{Journal of North American Benthological}

Society, v. 6, n. 42, p. 315-342. 1996.

AYRES-PERES, L.; SOKOLOWICZ, C. C.; SANTOS, S. Diversidade e abundância da macrofauna bentônica em ambientes lóticos da região central do estado do Rio Grande do Sul, Brasil. Biota Neotropica, v. 6, n. 3, p. 1-11. 2006.

BISPO, P. C.; OLIVEIRA, L. G. Distribuição espacial de insetos aquáticos (Ephemeroptera, Plecoptera, Trichoptera) em córregos de cerrado do Parque Ecológico de Goiânia, Estado de Goiás. In: NESSIMIAN, J. L., CARVALHO, A. L. (eds). Ecologia de Insetos Aquáticos. Rio de Janeiro: PPGE-UFRJ, Series Oecologia Brasiliensis, v. 5, 1998. p. 175-189.

BLANCO-BELMONTE, L. Estudio de las comunidades de invertebrados asociados a las macrofitas acuáticas de tres lagunas de inundación de la sección baja del Río Orinoco, Venezuela. Memoria de la Sociedad de Ciencias Naturales La Salle, v. 49-50 (133- 
134), p. 71-107.1989/1990.

BOYD, C. E. Amino acid, protein and caloric content of vascular aquatic macrophytes. Ecology, v. 51, p. 902-906. 1970.

BROWN, A.V.; BRUSSOCK, P.P. Comparisons of benthic invertebrates between riffles and pools.

Hydrobiologia, v. 220, p. 99-108. 1991

BUENO, A. A. P.; BOND-BUCKUP, G.; FERREIRA, B. D. P. Estrutura da comunidade de invertebrados bentônicos em dois cursos d'água do Rio Grande do Sul, Brasil. Revista brasileira de Zoologia, v. 20, n. 1, p. 115-125. 2003.

CALLISTO, M.; GOULART, M.; BARBOSA, F.R.A.; ROCHA, O. Biodiversity assessment of benthic macroinvertebrates along a reservoir cascade in the lower São Francisco river (Northeastern Brazil). Brazilian Journal of Biology, v. 65, n. 2, p. 229-240. 2005.

CARVALHO, A. L; CALIL, E. R. Chaves de identificação para famílias de Odonata (Insecta) ocorrentes no Brasil, adultos e larvas. Papeis Avulsos Zoologia, v. 41, n. 15, p. 223-241. 2000.

CHAMBERS, P. A.; LACOUL, P.; MURPHY, K. J.; THOMAZ, S. M. Global diversity of aquatic macrophytes in freshwater. Hydrobiologia, v. 595, p. 9-26. 2008.

CORBI, J. J.; TRIVINHO-STRIXINO, S. Spatial and bathymetric distribution of the macrobenthic fauna of the Ribeirão das Anhumas reservoir (Américo Brasiliense-SP, Brazil). Acta Limnologica Brasiliensia, v. 14, p. 35-42. 2002.

CORREIA, L. C. S. Comparação entre as assembléias de macroinvertebrados associados a substratos naturais e artificiais na represa do Monjolinho (São Carlos-SP). 1999. p. 78.
Dissertação (Mestrado em Ecologia e Recursos Naturais) - UFSCar, São Carlos, 1999.

DUDGEON, D. The influence of riparian vegetation on macroinvertebrate community structure in four Hong Kong streams. Journal of Zoology (London), v. 216, p. 609-627. 1988.

ESTEVES, F. A. Fundamentos de Limnologia. 2. Ed. Rio de Janeiro: Interciência, 1998. 602 p.

FERNÁNDEZ, H. R.; DOMINGUEZ, E. Guia para la determinación de los artrópodos bentônicos sudamericanos. San Miguel de Tucumán: Universidade de Tucumán. 2001. 282 p.

GLOWACKA, I.; SOSZKA, G. J.; SOSZKA, H. Invertebrates associated with macrophytes In: E. Pieczynska (ed.). Selected problems of lake littoral ecology. Warszawskiego, Warszawa: Widawnictwa Uniwersytetu Warszawskiego, 1976. $238 \mathrm{p}$.

HAMMER, O.; Harper, D. A. T.; Ryan, P. D. PAST: Paleontological statistics software package for education and data analysis. Palaeontologia Electronica, v. 4, p. 1-9. 2001.

HAUER, F. R.; RESH. V. H. Benthic macroinvertebrates. In: HAUER, F. R.; LAMBERT, G. A. Strean Ecology. San Diego: Academic Press, 1996. p. 339-369.

HYNES, H. B. The ecology of running waters. Canada: University of Toronto Press, 1970.555 p.

LAMBERTI, G. A.; MOORE, J. W. Aquatic insects as primary consumers, In: RESH, V. H. \& ROSENBERG D. M. (eds.). The ecology of aquatic insects. New York: Praeger Publishers, 1984. $625 \mathrm{p}$.

LAKE, P. S. Disturbing lard and soft bottom 
communities a comparison of marine and freshwater environments. Australian Journal of Ecology, v. 15, p. 477-488. 1990.

MARGALEF, R. Limnologia. Barcelona: Ed. Omega, 1983. 1010 p.

MASTRANTUONO, L. Community structure of the zoobentos associated with submerged macrophytes in a eutrophic Lake Nemi (Central Italy). Bollettino di Zoologia, v. 53, p. 41-47. 1986.

MCCFFERTY, W. P. Aquatic entomology. Boston: Jones and Bartlett Publishers, 1981. 448 p.

MERRITT, R.W.; CUMMINS, K.W. An introduction to the Aquatic Insects of North America. Dubuque: Kendall/Hunt publishing Co., 1996. $862 \mathrm{p}$.

MINSHALL, G.W., Aquatic insect-substratum relationships, In: RESH, V. H. \& ROSENBERG, D. $M$. (eds.). The ecology of aquatic insects. New York: Praeger Publishers, 1984. p. 358-400.

NIESER, N.; MELO, A. L. Os Heteropteros aquáticos de Minas Gerais. Guia introdutório com chave de identificação para as espécies de Nepomorpha e Gerromorpha. Belo Horizonte: Editora UFMG, 1997. 177 p.

OLIVEIRA, L. G.; BISPO P. C.; SÁ, N. C. Ecologia de comunidades de insetos bentônicos (Ephemeroptera, Plecoptera e Trichoptera) em córregos do parque ecológico de Goiânia, Goiás, Brasil. Revista Brasileira de Zoologia, v. 14, p. 867-876. 1997.

OLIVEIRA, L. G.; FROEHLICH, C. G. Natural Hystory of three Hydropsychidae (Trichoptera, Insecta) in a "Cerrado" stream from northeastern São Paulo, Brazil. Revista Brasileira de Zoologia, vol. 13, n. 3, p. 755-762. 1996.

PEIRÓ, D. F; ALVES, R. G. Insetos aquáticos associados à macrófitas da região litoral da represa do Ribeirão das Anhumas (Município de Américo Brasiliense, São Paulo, Brasil). Biota Neotropica, v. 6, n, 2. p. 1-9. 2006.

PEIRÓ, D. F; GORNI, G. R. Diferença na composição da entomofauna aquática associada a duas plantas de hábitos diferentes em um tanque de piscicultura. Multiciência, v. 10, p. 149-160. 2010.

RESH, V. H.; ROSEMBERG, D. M. The ecology of aquatic insects. New York: Praeger Publishers, 1984. $625 \mathrm{p}$.

ROSINE, W. N., The distribution of invertebrates on submerged aquatic plant surfaces in muskee lake, Colorado. Ecology, v. 36, p. 308-314. 1955.

SAULINO, H. H. L.; CARACCIOLI, L. C.; CORBI, J. J. Avaliação da qualidade da água da nascente do Ribeirão das Anhumas (Araraquara SP) através do estudo de macroinvertebrados aquáticos. Revista Uniara, vol. 14, n. 1, p. 17-29. 2011.

SONODA, K. C. Chironomidae (Díptera) da fitofauna de Cabomba piauhyensis. 1999. Dissertação (Mestrado em Ecologia e Recursos Naturais) - UFSCar, São Carlos. 1999. 60 p.

THOMAZ, S. M.; DIBBLE, E. D.; EVANGELISTA, L. R.; HIGUTI, J.; BINI, L. M. Influence of aquatic macrophyte habitat complexity on invertebrates abundance and richness in tropical lagoons. Freshwater biology, v. 53, p. 358-367. 2008.

THOMAZ, S. M.; CUNHA, E. R. The role of macrophytes in habitat structuring ins aquatic ecosystem: methods of measurements, causes and 
consequences on animal assemblage's composition and biodiversity. Acta Limnologica Brasiliensia, v. 22, n. 2, p. 218-236. 2010.

STRIXINO, G; TRIVINHO-STRIXINO, S. Macroinvertebrados associados a tapetes flutuantes de Eichhornia crassipes (Mart) Solms, de um reservatório. In IV Seminário Regional de Ecologia, São Carlos: Ed. UFSCAR, p. 375-392. 1984.

TRIVINHO-STRIXINO, S.; STRIXINO, G. Estrutura da comunidade de insetos aquáticos associados à Pontederia lanceolata nuttal.
Revista Brasileira de Biologia, v. 53, p. 103-111. 1993.

UIEDA, V. S.; GAJARDO, I. C. S. M.

Macroinvertebrados perifíticos encontrados em poções e corredeiras de um riacho. Naturallia, v. 21, p. 31-47. 1996.

WARD, J. V. Aquatic Insect Ecology. New York: Wiley \& Sons, 1992. 438 p.

WIGGINS, G. B. Larvae of the North American Caddisfly Genera (Trichoptera). Toronto: University of Toronto Press, 1977. 401 p. 